Publicación semestral. ISSN 2215-4906

Volumen 79 - Número 2

Enero - Junio 2020

\title{
Transfeminidades: el grito cuir y la performatividad afectiva
}

\author{
Transfeminities: the Queer Scream and the \\ Affective Performativity
}

Marusia Pola Mayorga

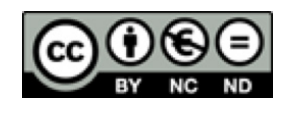

Esta obra está bajo una licencia Creative Commons Reconocimiento-No comercial-Sin Obra Derivada 


\title{
Transfeminidades: el grito cuir y la performatividad afectiva
}

\author{
Transfeminities: the Queer Scream and the \\ Affective Performativity
}

\author{
Marusia Pola Mayorga ${ }^{1}$ \\ Universidad Nacional Autónoma de México \\ México
}

Recibido: 30 de marzo de 2019 Aprobado: 03 de julio de 2019

\begin{abstract}
Resumen
Partiendo de una metodologia potenciada desde la musicología cuir, con c, en el siguiente articulo reflexiono sobre la estetización del cuerpo queer y el potencial expresivo de los afectos desde el trabajo y la música de tres artistas trans mexicanas. El imaginario sonoro desde donde construyen sus proyectos musicales me permite reflexionar sobre los afectos y las sensibilidades que entran en constante diálogo y negociación y que operan desde un exceso estético donde lo simbólico, lo auténtico y lo vernáculo están en constante dialogo artístico y epistémico.

Palabras clave: musicología cuir; grito; performatividad afectiva; performance; identidad; trasfeminidad

Abstract

Based on a cuir methodology, with c, in the following paper I reflect on the aesthetics of the queer body and the expressive potential of the affections from the work and music of three Mexican trans artists. The sound imaginary from where they build their musical projects, allows me to reflect on the affections and sensibilities that came into constant dialogue and negotiation. The aesthetic excess show in their performances evoke an artistic and epistemological dialogue where the symbolic, the authentic and the vernacular coexist.
\end{abstract}

Keywords: cuir musicology; scream; affective performativity; performance; identity; transfeminity

1 Estudiante del Doctorado en Música y Musicología en la Universidad Nacional Autónoma de México. Master of Music and Musicology por la Texas Tech University. Correo electrónico: pmarucia@hotmail.com

ESCENA. Revista de las artes, 2020, Vol. 79, Núm. 2, pp. 214-225 ISSN 2215-4906 


\section{El giro cuir}

La música es herramienta expresiva para la articulación de subjetividades agenciadas por los sujetos que se discursan a través de ella. En estos procesos creativos que se construyen entre el sujeto creador, el público y los espacios que interpela, me interesa especialmente la forma en que se construyen discursos, en los cuales se expresan y potencian el género y las identidades sexo-disidentes consideradas transgresoras. Desde el pensamiento de Butler (2006) y la queer theory, la identidad sexual es una ilusión retroactivamente creada por un performance que, a su vez, está afectado por el acontecer de convenciones y normas sociales que excluyen sistemáticamente a otras experiencias. Queer entonces, se vuelve una rúbrica politizada que apunta hacia la multiplicidad de género y una sexualidad vista desde una complejidad fluida que se significa desde un rango de subjetividades no normativas, prácticas e interrelaciones asociadas a un deseo no normado y que están en constante desafío con códigos morales impuestos por las sociedades dominantes.

En la queer musicology, el campo de análisis musical también se ha potenciado desde estas preocupaciones y se ha enfocado en cómo la música se vuelve un mecanismo expresivo de significación de género y sexualidad, capaz de despertar, canalizar e inscribir impulsos sexuales y deseos. Desde la musicología, las asociaciones con la queer theory, no solo han sido de carácter académico, sino también políticos en pos de construir desde una multiculturalidad que, al desprenderse de las nociones definidas desde categorías homogéneas contenidas en la disciplina, encuentra en lo queer un movimiento post-identitario que nombra a los cuerpos, las disidencias sexuales y los discursos estéticos desde la diversidad de prácticas desde donde se proyectan.

Si bien, la música ha sido ampliamente teorizada y analizada, desde la queer musicology, el cuerpo teórico ha sido construido desde narrativas y corpus de estudio erigidos, mayoritariamente, desde una academia anglófona que ha fallado en representar y analizar las dimensiones desde donde se proyectan sujetos que se discursan desde otras latitudes, prácticas y devenires históricos. Las mismas problemáticas que surgen en tono a la queer musicology, se presentan al hablar de lo queer desde Hispanoamérica y devela una urgencia, no de traducción, pero sí de desplazamiento del término hacia realidades latinoamericanas. En este espíritu, la posibilidad de una musicología cuir, con c, potencia la disciplina hacia el impulso de construir discursos que hagan frente al reto que constituye la creación de marcos metodológicos y aparatos críticos que se sitúen desde las voces y la experiencia Latinoamericana, y hagan frente a los modos heterogéneos y excluyentes heredados de una visión más positivista y euro centrada. La traducción pone en circulación el término en contextos de habla hispana y le otorga un peso lingüístico desde donde se reafirma su capacidad de subversión, al mismo tiempo, que disputa el supuesto copyright anglófono que puede evitar la reconfiguración epistémica de los estudios cuir. Valencia (2015) afirma: 
El uso del término queer y su derivación en cuir no obedece a un entusiasmo ingenuo -que hunde sus raíces en los deseos de legitimación a través del consumo cultural y la exportación de contenidos-, sino que su intención es tender puentes transnacionales de identificación y afinidad que reconozcan y visibilicen la vulnerabilidad históricamente compartida, entre los procesos de minoración -que emergieron como protesta critica en el tercer mundo estadounidense- a través de las multitudes queer con los procesos de subalternización histórica que se implantaron en nuestros territorios a partir de la colonización y nuestros propios devenires minoritarios (Valencia, 2015, p. 33).

Nombrar una musicología cuir, por tanto, no solo supone una mera actualización del término, sino que se opone a una asociación que pueda ser vista como un mero intento de validación académica que derive en una importación metodológica y epistémica. El giro epistémico que produce la traducción representa una desvinculación del término anglo al mismo tiempo que registra la inflexión geopolítica hacia al sur, entendido este como el espacio epistémico que da cuenta de experiencias otras encarnadas en sujetos atravesados por marcas coloniales y de dominación. En este sentido Valencia considera que la traducción funciona como una contraofensiva a la epistemología colonial y a la historiografía anglo-americana y fomenta la construcción de una teoría marcada por procesos donde se reconozcan las genealogías de pensamiento que han dado forma a la musicología, al mismo tiempo que se construyen y problematizan desde realidades hispanoamericanas, no para reclamar un uso que pueda ser entendido desde el sectarismo disciplinar, sino, para abrir la discusión e inclusión de epistemologías acotadas desde la condición histórica que suponen sujetos colonizados (2015, p. 34).

Esta discusión se origina no con el propósito de establecer espacios subdisciplinarios que pretendan volver a "capturar" en marcos epistémicos y discursos académicos los fenómenos musicales que puedan ser suscritos a nociones "latinoamericanistas" que sigan reproduciendo alteridad y hablando desde una diferencia casi esquizofrénica². La musicología cuir con c, funciona como un posicionamiento político que me da herramientas para reimaginar los procesos y los modos en que las rupturas sexo-disidentes negocian y reconfiguran el cuerpo como herramienta estética donde el exceso y lo simbólico están en constante cruce y construcción.

2 Una alteridad fetichizada desde ese falso "redescubrimiento" de la precariedad cultural del otro al que se piensa como incapaz de habitar los espacios discursivos sin pertenecer al conjunto de voces globales. Esta apropiacion de la "alteridad" produce a su vez procesos de hibridación que existen como fisuras a los sistemas hegemonicos aparentemente infranqueables (Barriendos Rodriguez, 2007).

ESCENA. Revista de las artes, 2020, Vol. 79, Núm. 2 (enero-junio), pp. 214-225 
En la siguiente parte reflexiono sobre estos procesos a partir del trabajo de tres artistas trans mexicanas y sus proyectos musicales, por lo cual es importante acotar las diferentes definiciones de transfeminidad y transfeminismo que suscribo en mi análisis.

\section{Transfeminidades}

Trans es un sufijo que se usa usualmente para describir personas que transgreden las normas de género o que dislocan la asignación biológica de este, cuando hablo de transfeminidad, la evoco desde el pensamiento de Emi Koyama y del manifiesto transfeminista. Koyama (2003) define la transfeminidad como la cualidad a la que se adscriben las identidades que se identifican, viven y se re-apropian de sensibilidades y características típicamente asociadas a lo femenino, dislocándose de la idea del sexo biológico. Sin embargo, Koyama también reconoce en el sufijo, el potencial de adscripción de cualquier identidad que transgreda la dicotomía hombre/mujer y que transite entre géneros y entre sus fronteras (Koyama, 2003).

También, lo trans funciona como un posicionamiento lingüístico que invita al lector a de-construir los significantes que componen el termino y que lo construyen desde una polisemia asociada también, al espíritu transgresor que define la investigación. Transfeminidad se construye a partir del sufijo trans ${ }^{\star}$, con asterisco, usado por Jack Halberstam en su libro del 2017 Trans: A Quick and Quirky Account of Gender Variability, Halberstam (2017) acota:

The asterisk modifies the meaning of transitivity by refusing to sitúate transtion in relation to a destination, a final form, a specific shape, or an established configuration of desire and identity. The asterisk holds off the certainty of diagnosis; it keeps at bay any sense of knowing in advance what the meaning of this or that gender variant form may be, and perhaps most importantly, it makes trans* people the authors of their own categorizations [El asterisco modifica el significado de transitividad al negarse a situar la transición en relación con un destino, una forma final, una forma específica o una configuración establecida de deseo e identidad. El asterisco retiene la certeza del diagnóstico; mantiene a raya cualquier sensación de saber de antemano cuál es el significado de esta o de la variante de género, y quizás lo más importante, hace que las personas trans* sean los autores de sus propias categorizaciones] (Halberstam, 2017, p. 12).

Halberstam usa el asterisco para desvincular el término de una relación transitiva que supone, un destino final, un significante fijo. Esta reconfiguración lo transforma en una categoría (umbrella term) potenciada y definida por los sujetos que deciden contenerse en ella. El sufijo supone entonces, una negación a las categorizaciones predeterminadas y se convierte en una herramienta epistémica sometida a la constante actualización y reconfiguración por parte de los sujetos que decidan definirse en ella. Retomo también el potencial 
del sufijo trans desde la metodología transdisciplinar propuesta por Barsarab Nicolescu (2010) donde establece la transdisciplinariedad como el devenir histórico de los procesos analíticos inicialmente propuestos la por multi y la interdisciplinariedad. Diferenciando estas de la transdisciplinariedad, por su naturaleza trascendental que la coloca entre disciplinas, a través de estas y más allá de estas al proponer un entendimiento del mundo por medio de un conocimiento relacional que está en dialogo y revisión permanente.

La transfeminidad está también ligada al transfeminismo como el devenir de las luchas feministas que repolitiza el cuerpo y los feminismos de la disidencia sexual. Utilizar el potencial del sufijo trans sin perder la nomenclautra del feminismo conserva las cualidades transitorias que contempla el desplazamiento de sujetos atravesados por distintas intersecciones y defiende formas de representacion "otras" capaces de articular los diferentes disensos del cuerpo. Lo trans tiene la cualidad de potenciar las identidades fuera de las dicotomías modernas y desarrollar nuestro análisis más allá del tránsito o el uso múltiple de marcos metodológicos y nos invita a pensar nuestro aparato critico desde las realidades que lo evocan y lo nombran.

\section{El grito cuir}

En mi interés por la creación de nuevos focos metodológicos irruptores, trans-disciplinarios e integrales, empecé a pensar en articular mis reflexiones desde una categoría que tuviera como principal característica la no contención y pudiera potenciar las cualidades estéticas de los sujetos o casos de estudio desde este primer objetivo. La no contención como característica de una categoría es en sí, una contradicción, sin embargo, si una de las principales preocupaciones de la epistemología es la naturaleza, tipología y taxonomía de las manifestaciones de los sujetos de estudio y de la relación de estos con el mundo, la potencia epistemológica de la creación de una categoría que me permita identificar, evaluar las fuentes y su fuerza es el primer principio con el que inicio mis indagaciones estéticas. La construcción del fenómeno al que, por ahora, llamo "Grito cuir" pasará de ser un objeto abstracto a un proceso que me permitirá plantarme frente a una teorización decantada a partir de su propia naturaleza y de los casos de estudio, el grito cuir es, entonces, la no-categoría desde donde hago mis primeras indagaciones.

Al aplicar un giro cuir, al corpus musical que me compete, hecho mano también de las reflexiones hechas por Judith Butler donde profundiza sobre la re-significación del termino queer y el poder performativo evocado por la subversión del lenguaje, Butler (1993) discute lo queer desde el poder político que se reivindica al posicionar el termino desde la indagación histórica y epistémica de los sujetos a los que nombra. Estas premisas me llevan a trabajar las músicas desde los imaginarios y espacios culturales que se subvierten a partir de las rearticulaciones propuestas por los artistas (Butler, 1993, p 170).

ESCENA. Revista de las artes, 2020, Vol. 79, Núm. 2 (enero-junio), pp. 214-225 
Las subjetividades proyectadas por los creadores, a menudo operan desde sensibilidades estéticas enunciadas desde muchos lados, esa aparente inteligibilidad con la que los cuerpos queer evocan sus sexualidades, pasiones e identidades, se convierte en voz, en un grito que se expande potencialmente para quebrar parámetros culturales predeterminados asociados a patrones dominantes donde, según Ochoa Gautier (2015), impera una modernidad plagada de silencio, desigualdad y desencuentros (Ochoa Gautier, 2015). De acuerdo con Barthes (1977) esa inmaterialidad del sonido, que se potencia poderosa y resonante, mantiene cualidades vinculantes y espaciales que permite a los cuerpos disidentes apropiarse de los espacios, reales y simbólicos (Barthes, 1977, p. 188).

Desde una definición clásica del Real diccionario de la lengua española, el grito se define como una voz muy esforzada y levantada que se alza vehementemente para expresar un sentimiento individual o colectivo. Dichas características típicamente asociadas al grito como fenómeno físico, se abstraen al ocurrir el grito queer y operan a nivel estético. El grito queer, entonces, sucede desde la performance musical y parece mantener al objeto sonoro en un estado de liminalidad latente en donde lo "real" y lo simbólico están en constante intercambio sin realmente pertenecer a ninguno y desde donde se agencia un exceso estético inscrito desde una performatividad queer destinada a irrumpir el discurso musical, entendiendo por performatividad a la creación de fenómenos en el momento mismo de la performance y que contienen un potencial transformativo del espacio en donde sucede (López Cano, 2008, p. 3).

Visto como un evento aural, dicha intencionalidad le otorga al grito una carga dramática y la potencialidad para apropiarse del espacio donde sucede el grito, por lo tanto, interpela a los sujetos a los que se dirige, ¿pero entonces, quién grita y por qué grita? Y ¿A qué sensibilidades o necesidades está respondiendo cuando el grito es potenciado como un acto aural performativo? Los fenómenos que asiento dentro de este imaginario teórico, comparten características que me permiten discursarlos desde estas cualidades. Los gritos, en su hábitat natural, demandan una respuesta directa, están pensados para ser una expresión irreprimible que quiere comunicar algo inmediato, sin embargo, el grito como fenómeno artístico o estético, resuena de manera diferente. Su sonoridad pone el énfasis y amplitud en el afecto y en la fuerza retórica de lo que agencia. El grito entonces puede ser enérgico o apenas un murmullo, puede estar físicamente o no, puede ser silencio o apenas intención, su fuerza retórica y estética es la que le permitirá alcanzar distancias y ser escuchado desde su urgencia. El grito cuir, por lo tanto, es trans femenino, trans disciplinar y trans musical. 


\section{Luisa Almaguer}

Conocí a Luisa Almaguer en un Conversatorio, organizado el pasado 1 de septiembre del 2017 por el colectivo Heterodoxias y dedicado a trazar puentes de encuentro entre los fenómenos musicales asociados a la nueva música urbana, transgresiones y nueva electrónica. El tema del conversatorio fue sobre la escena trans en la Ciudad de México y sus proyectos musicales. Luisa Almaguer fue una de las invitadas encargadas de cerrar el evento con un pequeño concierto al final, si bien su participación en el conversatorio fue de las más energéticas, con una agenda feminista muy clara y aguerrida lo que más me sorprendió fue su actuación. La fuerza de su voz y las técnicas con que la despliega, disloca al espectador y lo sumerge en un drama performativo en donde Luisa se confiesa en letras que hablan sobre amor y dolor. Las meta-narrativas de la voz de Luisa nos hablan de la relación personal que ella tiene con la misma y despliegan las distintas maneras en cómo funciona una voz situada desde una identidad cuir.

Además, Luisa habla de una lucha personal por aceptar una voz que no correspondía con la imagen que ella tenía de sí misma (L. Almaguer, comunicación personal, 24 enero del 2018). Luisa habla de cómo su voz se convirtió en una especie de enemiga que la delataba frente a un mundo acostumbrado a categorizar el género desde las más simples dicotomías. "Yo sé que puedo pasar como mujer mujer, el problema está cuando abro la boca" (L. Almaguer, entrevista personal, 24 enero del 2018). En su lucha por aceptar una voz que la ponía constantemente en un estado de vulnerabilidad, es en la música en donde ella encuentra la forma de conciliarse consigo misma.

Jarman-Ivens (2011) nos habla de las voces cuir y las maneras en que develan nociones de poder asociadas a la escucha y a la producción de las mismas (Jarman-Ivens, 2011, pp. 95-96). Jarman-Ivens (2011) discute la posibilidad de encontrar en las dislocaciones tímbricas y texturales las distintas formas en que opera una escucha y una evocación cuir. En el caso de Luisa, su voz se convierte en el foco de su grito cuir, el espacio en el que su performance musical sucede, se convierte confesional, sus letras no son políticas sin embargo los afectos contenidos en ellas si lo son, sus confesiones personales hablan de una experiencia afectiva trans, en la cual el dolor y el amor confluyen en un synth-pop oscuro que se delinea desde su voz de barítono.

Las vocalizaciones de Luisa ponen la atención del escucha en su timbre, un timbre grave y profundo que se presenta claro y libre de impostaciones. La performance musical de Luisa pone a consideración la voz como herramienta expresiva en donde la identificación y el deseo convergen alrededor del fenómeno cuir, los afectos y sensibilidades se convierten en potencial expresivo al transgredir desde una escucha que nos obliga a desarticular y desnaturalizar las nociones de lo femenino desde una voz que se de-construye y construye desde otras lógicas. 


\section{Alda Arita}

El rock y el jazz son algunas de las escenas musicales donde más se ha discutido la falta de inclusión y de representación y problematizado los discursos históricos y canónicos desde donde se erigen. La música de Alda transita, principalmente entre estos dos géneros, aunque su proyecto musical parece adscribirse también a múltiples escenas donde podemos escuchar evocaciones de noise, folk y experimentación. La guitarra parece ser su instrumento principal, aunque Alda se confiesa multiinstrumentista. Alda define su lenguaje musical a través de su proceso como mujer trans, su técnica musical rechaza modelos genealógicos de producción que contextualizan el género de rock y el de jazz desde estéticas patriarcales relacionadas con un legado virtuosistico y una continuidad "canónica".

El lenguaje de Alda se desprende de estos discursos y es a partir de la improvisación libre, la experimentación, el uso de múltiples instrumentos como la trompeta, el citar y diversas tecnologías musicales y el trabajo colaborativo desde el cual construye su universo musical.La música de Alda es pausada, pero enérgica; sus improvisaciones están llenas de sonidos atmosféricos que va construyendo durante el performance musical al mismo tiempo que su guitarra evoca riffs y melodías donde el lenguaje del rock, el punk, el folk, el jazz y el noise confluyen libremente. En entrevista realizada en Radio UNAM (2017), Alda habla de cómo la improvisación le permite abstraerse de las lógicas imperantes y le da oportunidad de entrar en un estado casi de trance desde donde explora las posibilidades de la intuición y el potencial de los afectos expresivos (Alda Arita citada en Sorroza, junio de 2017).

En esta ocasión, el proyecto en el que me enfoco es una colaboración de spoken word e improvisación libre entre Alda y la poetisa mexicana Edmeé García alias la diosaloca. El espacio sónico creado por las dos artistas sitúa la escucha desde la efimeralidad del sonido y nos coloca en medio de una conversación que trasciende la semántica y la gramática y nos alienta como escuchas a participar en un proceso de re significación donde escucha y sonido exceden las imposiciones aurales. La poesía de diosaloca me recuerda los trabajos literarios de Gertrude Stein en donde se exploraban los límites de la acción comunicativa y el lenguaje representacional. Chani Marchiselli (2016), analiza las implicaciones feministas de los dispositivos sónicos de Gertrude Stein y los posiciona desde un paralenguaje creado por Stein en donde la fonética y los significantes nacidos a partir de una lectura en voz alta potencian sonoridades que agencian colaboración colectiva y la posibilidad de una sonoridad cuir desde donde también se discuten nociones de domesticidad y estrategias comunicativas alternas (Marchiselli, 2016)

Si bien el trabajo de Marchiselli se centra en la manera en que la poesía de Stein obliga al escucha a volverse también lector y creador, encuentro similitudes al acercarme al spoken word de Alda y Edmeé. Al igual que el trabajo de Stein, el spoken word sugiere 
repensar el lenguaje convencional al mismo tiempo que problematiza las desigualdades producidas por el mismo. Edmeé y Alda juegan con la fonética y los sonidos al mismo tiempo que su poesía habla y grita:

Hablo, y escribo poesía porque quiero ser libre

Porque en este cuerpo limitado, en este lenguaje definido

Por los mecanismos de mi mente, vi el creyente del espacio

Tiempo y sus dimensiones, tengo mi respiración infinita, porque

Aun sumergida en una experiencia de sonidos que a veces me engañan

Tengo la marea de mi respiración infinita... respira poesía, respira...(García, 2016, 1:18').

\section{La Bruja de Texcoco}

La Bruja prepara su escenario casi de manera ritual. Su estudio está lleno de máscaras, instrumentos y ofrendas a la tierra, ella sentada en medio, ataviada con un huipil amuzgo de gala, el pelo largo engalanado con un rebozo como moño, usa tacones y esta cubierta de collares, su maquillaje es fuerte y colorido, apelando a la estética drag, ojos azules, labios rojos y una pesada y bien cuidada barba. La Bruja de Texcoco fue de las primeras artistas a las que me acerque al iniciar mi investigación doctoral. Me llamo la atención no solo la voluptosidad de su performance musical sino la particular re-apropiación que hacía de la música folclórica mexicana.

En su performance musical, la Bruja interviene su cuerpo apelando a un feminidad recargada, casi artificiosa, enmarcada en una estética camp donde el exceso evoca sensibilidades estilísticas asociadas a "Io tradicional mexicano", sin embargo, la Bruja retoma dichos elementos no desde su forma más clásica que ya, de por sí, levanta una serie de problemáticas asociadas a "lo tradicional", sino apelando a un estética que parece problematizar los símbolos y significantes de lo regional situándolos desde una expresión urbana. El exceso de lo mexicano pone en dialogo la contemporaneidad de saberes que se han discursado desde un pasado que se pretende conservar, pero que se excluye del discurso de desarrollo contemporáneo. La Bruja enmarca su personae musical desde la construcción de un performance donde una especie de "folckloricuir" sucede y es grito, y las poéticas y saberes vernáculos pertenecen, fluyen y se encarnan en la artista. 


\section{A modo de cierre}

Las evocaciones tanto afectivas como identitarias observadas en el performance de cada artista no funcionan como un mero accesorio, sino como una motivación y una reflexión epistémica desde donde la afectividad cuir es capaz de transformar y re-articular el espacio sónico desde el que se inscribe. Las afectividades disidentes se traducen en la experiencia musical que se define en el escenario, desde el punto de vista del giro afectivo, el potencial de los afectos y la emocionología permea la política y las formas en que los medio se relacionan con el sujeto, el potencial de una performatividad afectiva sitúa los espacios sónicos y los vuelve sociales y los afectos pre-personales, es decir, dirigidos a afectar para ser afectados. Al sumergirnos en un imaginario estético queer podemos observar modelos afectivos, sociales y comunitarios desde donde se produce material cultural que al estar en contradicción y en discordancia con las características estandarizadas de los modelos heteronormativos, los desestabilizan.

El trabajo de estas y muchos más artistas, se teje desde distintas líneas de pensamiento que potencian la construcción de un aparato crítico y de enfoques metodológicos imaginados desde las rupturas y los disensos del cuerpo. Una musicologia cuir nos invita a pensar en espacios epistemológicos que rompan las proxemicas erigidas desde la academia y observe las relaciones que se crean entre el artista creador, las audiencias y los espacios que se forman apartir de estas. Estos espacios de auralidad se convierten en lugares que nos brindan la oportunidad de explorar en detalle las posibilidades de una escucha que disloque nuestra propia percepcion y los modos en que producimos conocimiento a partir de ello. Las oportunidades de desidentificación entre actuantes y escuchas impulsa las subjetividades contenidas en cuerpos fronterizos cuya lectura siempre excede los disciplinamientos que supone su constante captura.

\section{Referencias}

Barriendos Rodriguez, J. (2007). El arte global y las políticas de la movilidad. Desplazamientos (trans)culturales en el sistema internacional del arte contemporáneo. LiminaR. Estuidos Soaicles y Humanísticos, 1, 159-182.

Barthes, R. (1977). Image, Music, Text. Hammersmith: Fontana Press.

Butler, J. (1993). Bodies That Matter: On the Discursive Limits of "Sex". New York: Editorial Routledge.

Butler, J. (2006). Deshacer el género. Barcelona: Editorial Paidos.

Chani Anine Marchiselli. (2016). Queer Sonorities: Sound as Persuasion in Gertrude Stein's Tender Buttons. Women's Studies in Communication, 39(1), 69-85. 
García, E. (2016). Respira poesia: 6 poemas en 3 lenguas. Recuperado de http://diosaloca. $\mathrm{mx} /$ respira-poesia-6-poemas-en-3-lenguas/

Halberstam, J. (2017). Trans: A Quick and Quirky Account of Gender Variability. Oakland: University of California Press.

Jarman-Ivens, F. (2011). Queer Voices: Technologies, Vocalitites and the Musical Flaw. New York: Palgrave Macmilan.

Koyama, E. (2003). The Transfeminist Manifesto. En R. Dicker y A. Pepmeir (Eds), Catching a Wave: Reclaiming Feminism for the Twenty-First Century (p. 244-261). Lebanon: University Press of New England.

López Cano, R. (2008). Performatividad y narratividad musical en la construcción social de género. Una aplicación al tango queer, Timba, Regetón y Sonideros. En Rubén Gómez Muns y Rubén López Cano (eds.), Música, ciudades, redes: creación musical e interacción social. Recuperado de https://dialnet.unirioja.es/servlet/libro?codigo=365912

Nicolescu, B. (2010). Methodology of Transdisciplinarity: Levels of Reality, Logic of the Included Middle and Complexity. En Transdisciplinary Journal of Engineering \& Science, 1(1), 19-38.

Ochoa Gautier, A. M. (2015). Silence. En D. Novak y M. Sakakeeny (eds.), Keywords in Sound (p. 183-192). Durham: Duke University Press.

Sorroza, M. (Junio de 2017). Entrevista y música en vivo con Alda Arita en Radio UNAM. Recuperado de https://soundcloud.com/aldaer/entrevista-y-musica-en-vivo-con-aldaarita-en-radio-unam

Valencia, S. (2015). Del Queer al Cuir: Ostranénie Geopolítica y Epistémica desde el Sur Glocal. En F.R Lanuza y R. M. Carrasco (eds.), Queer \& Cuir: Políticas de lo irreal (p. 19-37). México: Editorial Fotamara. 